\title{
HISTORIA Y PERFIL PROFESIONAL DE LA INSTRUMENTACIÓN QUIRÚRGICA
}

\author{
Jeannette Neira González*
}

\section{Resumen}

Desde sus comienzos en los años cuarenta hasta nuestros días la instrumentación quirúrgica ha recorrido un arduo camino para su profesionalización. El perfil del instrumentador quirúrgico formado en la Fundación Universitaria de Ciencias de la Salud es el de un profesional íntegro, conprometido con la particiáción comunitaria y con una sólida formación social, humanística y científica, de modo que puede desempeñarse en las áreas de administración, docencia, investigación, mercadeo, centrales de esterilización, salas de cirugía, suministro de materiales, hemodinamia y perfusión cardíaca.

\section{Antecedentes}

A mediados de la década de los cuarenta, cuando en Colombia comenzó el perfeccionamiento de la cirugía aplicando métodos de asepsia, los médicos no encontraban personal capacitado que integrara el equipo quirúrgico, para el manejo de los elementos necesitados en el acto quirúrgico y se presentaban grandes dificultades al buscar un elemento sepultado bajo un gran cúmulo de instrumental sucio de sangre, desordenado y poco estético en el quirófano, además de la pérdida de tiempo y la prolongación del acto operatorio debido a una causa mínima.

Es así como el doctor Juan Di Domenico se ofrece para instrumentar una de las operaciones gástricas del servicio. Por fortuna, el doctor Anzola operaba con mucho orden y previamente se hizo el planeamiento de los tiempos quirúrgicos a seguir y la insinuación al cirujano de no retirar la mirada del procedimiento, sino que extendiera la mano y le sería alcanzado el instrumental solicitado. Ante el asombro de cirujano, ayudante y asistente, todo procedió con precisión matemática.

Al terminar la operación, la mesa del instrumental quedó limpia y ordenada; la instrumentación fue todo un espectáculo, así como un verdadero éxito y en ese momento se puede afirmar, sin la menor duda, que nació la Escuela de Instrumentación del Hospital de San José, la cual marcó la pauta para que otros centros hospitalarios adoptaran las mismas medidas.

\footnotetext{
* Facultad de Instrumentación,, Fundación Universitaria de

Ciencias de la Salud, Bogotá, Colombia
}

Ante la necesidad de personal mejor calificado, en 1950, la señora Celmira Acevedo de Segura, enfermera egresada de la Universidad Nacional y quien acababa de realizar una especialización en Enfermería y Asepsia Quirúrgica en el hospital John's Hopkins de la ciudad de Baltimore, en Estados Unidos, presenta una proposición al presidente de la Sociedad de Cirugía de Bogotá del Hospital San José y a sus directivas.

Tal proposición fue acogida y se nombró una comisión bajo la dirección de la señora Helen Howilt, entonces Decana de la Facultad de Enfermería, para elaborar el primer programa de instrumentación quirúrgica, con el único fin de formar personal capacitado que colaborara en las técnicas quirúrgicas dentro del quirófano, asistiendo a los médicos en la labor de preparar los instrumentos, suturas, materiales y accesorios durante el acto quirúrgico.

\section{Recuento histórico}

El 23 de febrero de 1951, por decreto 402 de la Escuela Superior de Higiene, es aprobada la escuela de Instrumentación del Hospital San José, dirigida por la señora Celmira Acevedo de Segura; el curso tenía una duración de 18 meses distribuidos así: cuatro meses de enseñanza teórica, dos meses teórico-prácticos y doce meses de práctica intensiva.

Algunas alumnas que ingresaron al Hospital San Juan de Dios, crearon allí una nueva escuela de instrumentación, basadas en la experiencia del Hospital de San José. En 1954 se gradúa el primer grupo de instrumentadoras 
de esta escuela y con posterioridad se tomó tal fecha como el día nacional de la instrumentadora quirúrgica.

Años más tarde, el 19 de julio de 1979, mediante resolución 1188 se reconoce la carrera como técnico-quirúrgica; de igual modo, mediante la ley 6 del 14 de enero de 1982, es reglamentada la profesión tecnológica en instrumentación quirúrgica.

En 1989, se crea la Fundación Tecnológica de Carreras Paramédicas, conformada por las Escuelas de Instrumentación Quirúrgica y de Enfermería, con personería jurídica del Ministerio de Educación Superior y resolución 3836 de mayo de 1989.

El 26 de agosto de 1992, se aprueba el programa de Tecnología en Instrumentación Quirúrgica, mediante resolución número 002040, con una duración de seis semestres. La primera promoción de egresadas se graduó el 7 de julio de 1993.

El ideal de todas las facultades que actualmente se hallan en el ámbito técnico y tecnológico es lograr la profesionalización de la carrera y para ello se trabaja en conjunto con ACFIQ (Asociación Colombiana de Facultades de Instrumentación Quirúrgica). Así, mediante el acuerdo 12, del 12 de julio de 1996, se crea el programa de Profesionales en Instrumentación Quirúrgica.

En la actualidad se está presentando un proyecto de ley con el objetivo de reglamentar la profesión de Instrumentación Quirúrgica y actualizar la ley de Instrumentación Técnico-Quirúrgica, para proteger a los profesionales universitarios, quienes hasta el momento están desprovistos de legislación.

El interés, entonces, es ajustar dicha ley a la Constitución Política de Colombia, a la ley 30 de 1992 y a la ley 100 de 1993, para lo cual es preciso determinar la naturaleza, el propósito y el campo de aplicación de la instrumentación quirúrgica; además, es necesario establecer los principios que rigen el desempeño profesional de la instrumentación, señalar sus entes rectores de dirección, organización, acreditación y control del ejercicio profesional. Esta profesionalización permitirá, a su vez, que se abra el espacio para los postgrados y para las especializaciones en dicha disciplina.

Según el artículo 247 relacionado con el ofrecimiento de programas académicos en el área de la salud por parte de las instituciones de educación superior:

"Para desarrollar programas de pregrado o postgrado en el área de la salud, que impliquen formación en el campo asistencial, las instituciones de educación superior deberán contar con un centro de salud propio o formalizar convenios docente-asistenciales con instituciones de salud, que cumplan los tres niveles de atención médica, para poder realizar las prácticas de formación. En tales convenios se establecerán claramente las responsabilidades entre las partes..."

\section{Perfil profesional}

Para la Fundación Universitaria de Ciencias de la Salud (FUCS) el perfil del instrumentador quirúrgico que requiere el país es el de un trabajador íntegro, con conocimiento integral de la medicina, que sienta la obligación de la participación comunitaria y con una sólida formación social, humanística y científica. Lo anterior no excluye la adquisición de amplios conocimientos científicos y técnicos, inclusive aquellos que sólo pueden ser adquiridos en instituciones hospitalarias, o especializadas.

En cualquier campo en que el ser humano se ubique debe trazar su propia proyección. Toda profesión tiene como fin específico la contribución con el mejoramiento de la sociedad. El término proyección significa la aplicación de la profesión como un servicio social, junto con el empleo de las propias facultades en provecho del prójimo, con beneficio propio y recíproco.

Por otra parte, el bien común no puede ser ajeno a la existencia de la profesión hasta un interés particular; siendo la profesión la ocupación del hombre con un fin concreto y la aplicación ordenada y funcional de parte de la actividad humana a la consecución de cualquiera de los finales particulares, puede definirse que la proyección social es la voluntad de poner a contribución la propia personalidad en cada una de sus actuaciones y entregar, por completo, el desempeño a cada una de sus actividades.

El instrumentador quirúrgico, gracias a sus conocimientos teóricos y prácticos puede desempeñarse en los campos de: administración, docencia, investigación, mercadeo, centrales de esterilización, salas de cirugía, suministro de materiales, hemodinamia y perfusión cardíaca.

\section{Área administrativa}

Son los conocimientos básicos y preliminares de la administración en general con miras a brindar capacidad, de organización, logrando establecer un programa regular de capacitación y actualización permanente, que permita diseñar y aplicar estos conocimientos, especialmente en el campo de manejo de personal.

De igual modo, en el campo docente-administrativo en instituciones de educación superior, aportará todos y cada uno de los conocimientos y experiencias adquiridos dentro de la profesión. 


\section{Área docente}

Las funciones planteadas en esta área tiene como fin la introducción, capacitación y actualización del recurso humano en instrumentación, para lo cual desarrolla actividades investigativas, asistenciales, administrativas y educativas. Cuenta, además, con un intercambio profesional de otras disciplinas, con el fin de incrementar los conocimientos y orientar el ejercicio profesional.

Dentro del área en cuestión se deben elaborar planes de estudio que permitan capacitar al profesional con datos de actualidad, evitando caer en el estancamiento científico. También implica otras actividades como brindar asesoría académica intra y extra institucional, encaminada a actualizar al profesional, el estudiante y al personal del quirófano.

\section{Área de investigación}

Este núcleo formativo alcanzará su rumbo, si se enfoca dentro de los principios de "aprender a aprender" y "aprender por descubrimiento", es decir, que se desarrolla por medio de sentido crítico, ante el medio circundante y especialmente ante las fuentes de información de las que se extraen los conocimientos: documentos, medios de comunicación, libros, etcétera, para determinar en cada una de ellas su confiabilidad, credibilidad y validez. El área de investigación también busca y promueve la participación en estudios investigativos.

\section{Área de mercadeo}

El profesional de instrumentación, al afrontar el área de mercadeo, tiene como función promover, vender o dar facilidades para adquirir instrumental o equipo especializado, que se requiera en diversas cirugías y especialidades, además de materiales de suturas, elementos de esterilización y desinfección.

\section{Área de esterilización}

Tiene a su cargo la planeación y organización de los requisitos mínimos utilizados en ésta área, obteniendo controles permanentes que aseguren la prevención oportuna de infecciones, es decir que uno de sus conocimientos principales es la asepsia. Así mismo debe encaminarse al manejo adecuado de la esterilización y la manipulación de los diferentes equipos y la ropa que se debe utilizar durante los procedimientos.

\section{Área quirúrgica}

En este campo la instrumentadora quirúrgica tiene como fin la coordinación del funcionamiento eficaz del acto quirúrgico, asistiendo al cirujano con el manejo práctico del instrumental y los elementos necesarios para cada cirugía. Gracias a sus conocimientos teóricos y prácticos la instrumentadora prevé, planifica y cubre las necesidades de los otros miembros del equipo quirúrgico humano, a la vez que debe anticiparse a todas las posibles complicaciones del procedimiento.

\section{Área de suministros}

Tiene bajo su cargo la organización y pedido tanto de material de suturas como de instrumental, velando por su mantenimiento y la reposición del mismo. Para ello la instrumentadora participa en el comité de compras para la adquisición de elementos.

\section{Área de hemodinamia}

Su objetivo está encaminado a asistir al médico en los diferentes actos quirúrgicos donde se realicen procedimientos de diagnóstico y tratamiento, velando por el buen manejo del instrumental especializado y el cumplimiento de las normas asépticas.

\section{Área de perfusión}

Comprende el manejo de los diferentes equipos necesarios para procedimientos cardíacos de alta complejidad; específicamente la máquina extracorpórea y diferentes soluciones que son necesarias para el paciente, las cuales son manejadas por la instrumentadora quirúrgica.

\section{Conclusión}

Para quien haya entrado en la profesión llevado por una verdadera vocación y se ha encontrado a sí mismo en la práctica de su carrera, esta consagración constituye más que un deber, un placer espiritual. Si la profesión se vive, quedará tiempo para emplearlo en otras ocupaciones que de alguna manera disponen las facultades para un mayor y mejor rendimiento.

\section{Bibliografía}

1. ACITEQ. Pasado, presente y futuro de la instrumentación quirúrgica, 1996.

2. Di Domenico J. Colaboración con aporte de reseña histórica, 2000.

3. Artunduaga Montenegro GE, Gómez Méndez SP, Leguizamón Chaparro Y.Perfil profesional de la instrumentadora quirúrgica. 1997. 4. Aguilera S. Alvarez B, Avendaño N, Balamba D, Manso W, Paredes M. y col. Pasado, presente y futuro de la instrumentación quirúrgica. 1998.

5. Ramírez L, Moreno Y, Perdomo L, Peña M, Hernández H. Díaz A. Reseña histórica de la instrumentación quirúrgica en el Hospital de San José.

6. Cámara de Representantes. Ponencia de la Ley Número 086 de 1999. 